when systemic inflammation was inhibited by the non-steroidal anti-inflammatory drug (NSAID) meloxicam immediately after surgery, with the treatment ending 4 days before injection of D2A1-GFP cells, tumour growth in wounded mice was significantly reduced compared with that in untreated wounded mice. This effect was accompanied by an altered, less immunosuppressive phenotype of tumour-associated macrophages.

While this study shows that surgery and wound healing were positively linked with increased distant tumour growth in this model, addressing other outstanding issues such as the effect of surgery on disseminated tumour cells and other mechanisms of metastatic dormancy is important to fully understand the role of surgery in early relapse of breast cancer.

Ulrike Harjes

ORIGINAL ARTICLE Krall I $\mathrm{N}$. et al. The systemic response to surgery triggers the outgrowth of distant immune-controlled tumors in mouse models of dormancy. Sci. Transl Med. 10, eaan3464 (2018)

trees constructed from these features closely mirroring the mutation-based trees. A notable observation was that alterations in methylation and gene expression patterns in each cancer were heritable, stable and seemingly independent of the tumour microenvironment as these patterns were maintained despite growth in vitro.

Finally, treatment of clonal cancer organoids in vitro with chemotherapeutic agents and targeted therapies commonly used to treat colorectal cancer revealed striking variation in drug responses, even among closely related clones, and the presence of pre-existing resistant cells.

This study highlights the power of organoid technology to gain deeper insights into tumour evolution, perhaps leading the way for implementation of precision medicine.

Anna Dart

ORIGINAL ARTICLE Roerink, S. F. et al. Intratumour diversification in colorectal cancer at the single-cell level. Nature. https://doi.org/10.1038/ s41586-018-0024-3 (2018)

FURTHER READING Drost, J. \& Clevers, H.

Organoids in cancer research. Nat. Rev. Cancer

https://doi.org/10.1038/s41568-018-0007-6 (2018)

\title{
Inverted architecture
}

Mutation of histone $\mathrm{H} 3$ lysine 27 to methionine (H3K27M) is common in aggressive paediatric diffuse midline gliomas. This mutation alters epigenetic programmes, suggesting that cell differentiation might be altered; however, little is known about the cellular architecture of these tumours in patients.

To examine the cellular makeup of these tumours, Filbin, Tirosh, Hovestadt et al. used single-cell RNA sequencing (scRNA-seq) analyses of 2,458 cells derived from diagnostic biopsies of 6 patients with $\mathrm{H} 3 \mathrm{~K} 27 \mathrm{M}$ gliomas. They separated malignant from non-malignant cells and then compared the transcriptomes of H3K27M glioma cells with those they had previously identified in other glioma subtypes, including isocitrate dehydrogenase (IDH)-mutant gliomas (oligodendrogliomas and astrocytomas). Many genes were differentially upregulated in H3K27M gliomas, which is consistent with the role of $\mathrm{H} 3 \mathrm{~K} 27 \mathrm{M}$ in repressing the polycomb repressive complex 2 (PRC2).

Looking closer at the transcriptional programmes within the $\mathrm{H} 3 \mathrm{~K} 27 \mathrm{M}$ gliomas, the authors identified three differentiation states: astrocyte-like (AC-like), oligodendrocyte-like (OC-like) and oligodendrocyte precursor cell-like (OPC-like). The overlap between these programmes and cell cycle and stemness programmes indicated that AC-like and OC-like cells were largely quiescent and differentiated. Proliferation occurred predominantly in the OPC-like cells, which also were much more undifferentiated. Although the fraction of each cell population present in each tumour varied, OPC-like cells were consistently the most prevalent. This is interesting as it suggests that cancer stem cells do not necessarily have to be a rare population within a tumour.

Comparison of the hierarchies of H3K27M gliomas with IDH-mutant gliomas indicated that although the glioma subtypes express different genes, the observed subpopulations of cells are similar. However, although both glioma subtypes had AC-like, OC-like and stem or progenitor-like cells, there were many more cycling and undifferentiated cells in H3K27M gliomas, an observation that might explain the more aggressive behaviour of $\mathrm{H} 3 \mathrm{~K} 27 \mathrm{M}$ gliomas.

Further analysis of the different cell types in H3K27M gliomas indicated that OPC-like cells express target genes normally repressed by PRC2, but expression of these is lost in OC-like cells. Repression of these genes might be required for differentiation to an OC-like state; interestingly,

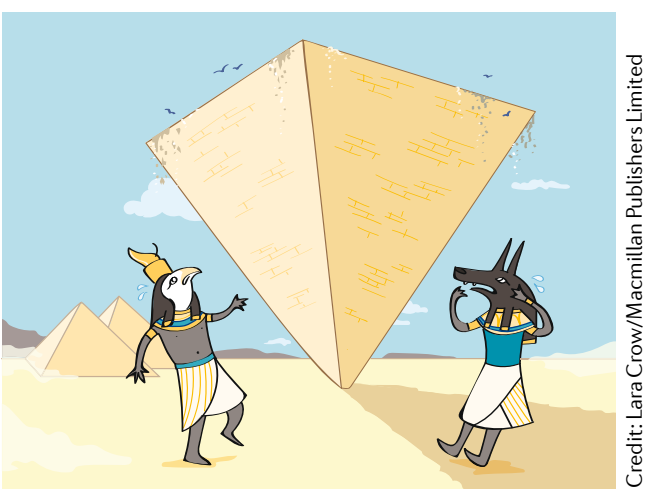

the PRC1 component BMI1 is upregulated overall in $\mathrm{H} 3 \mathrm{~K} 27 \mathrm{M}$ gliomas and might partially compensate for PRC2 loss to facilitate OPC-like to OC-like differentiation in some cells.

Furthermore, the OPC lineage marker platelet-derived growth factor receptor- $\alpha$ (PDGFRa) was highly expressed in OPC-like cells, and CRISPR-mediated knockout of PDGFRa reduced viability of patient-derived $\mathrm{H} 3 \mathrm{~K} 27 \mathrm{M}$ glioma cell lines. A BMI1 inhibitor further reduced viability of these cells. Therefore, targeting of PDGFRa and BMI1 might represent a viable therapeutic strategy for these tumours.

Gliomas, like many other tumours types, have substantial intratumour heterogeneity. The authors identified distinct genetic subclones in two H3K27M gliomas. Similar cell state hierarchies were observed within each subclone, suggesting that the cellular architecture is independent of subclonal somatic genetic alterations present in the tumour.

Overall, this scRNA-seq analysis indicates that aggressive $\mathrm{H} 3 \mathrm{~K} 27 \mathrm{M}$ gliomas, in contrast to IDH-mutant gliomas and normal development, seem to have an inverted cellular architecture in which undifferentiated, stem-like cells substantially outnumber differentiated cells. Furthermore, it suggests that lineage-based therapeutic targeting of PDGFRa might be beneficial for most $\mathrm{H} 3 \mathrm{~K} 27 \mathrm{M}$ gliomas regardless of additional genetic alterations.

Sarah Seton-Rogers

ORIGINAL ARTICLE Filbin, M. G., Tirosh, I., Hovestadt, V. et al.

Developmental and oncogenic programs in H3K27M

gliomas dissected by single-cell RNA-seq. Science $\mathbf{3 6 0}$

331-335 (2018)

FURTHER READING Tirosh, I. et al. Single-cell RNA-seq supports a developmental hierarchy in human oligodendroglioma. Nature 539, 309-313 (2016) |Venteicher, A. S. et al.

Decoupling genetics, lineages, and microenvironment in IDH-mutant gliomas by single-cell RNA-seq. Science 355 , eaai8478 (2017) 